\title{
Primary Central Nervous System Vasculitis Presenting with Isolated Headache
}

\author{
Ayush Agarwal ${ }^{1, \odot}$ Pradeep S. Reddy \\ ${ }^{1}$ Department of Neurology, All India Institute of Medical Sciences, \\ New Delhi, India \\ ${ }^{2}$ Department of Neuroradiology, All India Institute of Medical \\ Sciences, New Delhi, India
}

J Neurosci Rural Pract 2020;11:678-679

Primary central nervous system vasculitis (PCNSV) is an uncommon inflammatory disorder affecting small-tomedium-sized vessels of the central nervous system. ${ }^{1}$ Patients present with multiple symptoms of varying duration, severity and progression, headache, and cognitive dysfunction being the commonest followed by focal deficits, seizures, and ataxia. ${ }^{1}$ We present a PCNSV patient presenting with isolated headache.

A 22-year-old male, presented with progressively increasing, intermittent, holocranial headache, of moderate intensity, lasting for 2 to 4 hours, twice to thrice per week for past 2 years. This was not associated with photophobia/ phonophobia/nausea/vomiting/chemosis/lacrimation/ nasal congestion/variation with postural change. He had no other symptoms and mild responsive to nonsteroidal antiinflammatory drugs. Clinical examination was normal.

Routine investigations, vasculitic profile, viral markers, erythrocyte sedimentation rate (ESR), and C-reactive protein (CRP) were normal. Magnetic resonance imaging (MRI) with MR angiography brain (with contrast) revealed T2-FLAIR (fluid-attenuated inversion recovery) hyperintensities in left frontal lobe with susceptibility-weighted imaging (SWI) sequence showing multiple microbleeds ( - Fig. 1A, B). PCNSV was considered in view of cortical-subcortical microbleeds. Cerebrospinal fluid (CSF) examination showed five cells (100\% lymphocytes), $52 \mathrm{mg} / \mathrm{dL}$ glucose (random blood sugar: $76 \mathrm{mg} / \mathrm{dL}$ ) and $35 \mathrm{mg} / \mathrm{dL}$ protein. CSF cultures, malignant cytology and oligoclonal bands were negative. Cerebromeningeal biopsy was performed from the right temporal pole for tissue diagnosis, revealing findings suggestive of granulomatous type PCNSV ( Fig. 2A, B).
Address for correspondence Venugopalan Y. Vishnu, DM, Department of Neurology, All India Institute of Medical Sciences, Ansari Nagar, New Delhi 110029, India (e-mail: vishnuvy16@gmail.com).

He was treated with intravenous methylprednisolone pulse (1g/day for 5 days), followed by monthly cyclophosphamide infusions $\left(750 \mathrm{mg} / \mathrm{m}^{2}\right)$ for six doses and maintenance therapy with azathioprine $(100 \mathrm{mg} /$ day $)$. His headache improved and is asymptomatic at 2-year follow-up.

Diagnosis of PCNSV requires development of neurologic deficit unexplained by other processes, along with characteristic findings on angiogram, or CNS biopsy showing vasculitis. ${ }^{2}$ Our patient did not fulfil this criteria, but brain biopsy was diagnostic. Approximately $10 \%$ PCNSV patients are biopsy positive and angiography negative. ${ }^{1}$

PCNSV presentation with isolated headache (classified as 6.4.2 under the International Classification of Headache Disorders-3rd edition [ICHD-3] nomenclature ${ }^{3}$ ) has not been reported. Headache results from inflammation of vessels, leading to inflammatory cytokine release, which is relayed to the thalamus and limbic-parietal cortex leading to pain sensation. ${ }^{4}$

Presence of localized cortical-subcortical microbleeds, in absence of vascular risk factors, should raise suspicion of PCNSV. ${ }^{5}$ These are missed on routine MRI sequences, thereby highlighting the importance of SWI. ${ }^{5}$

Constitutional symptoms are usually absent and CSF examination is abnormal in 93\% cases. ${ }^{1}$ ESR and CRP are elevated in 17.5 and $33 \%$ patients, respectively. ${ }^{1}$ Both were normal in our patient. MRI brain is abnormal in $96 \%$ cases $^{1}$; infarcts being more common, unlike our patient.

We seek to highlight the importance of neuroimaging which led to the consideration of PCNSV as a differential diagnosis and the importance of achieving a tissue diagnosis via cerebromeningeal biopsy. 

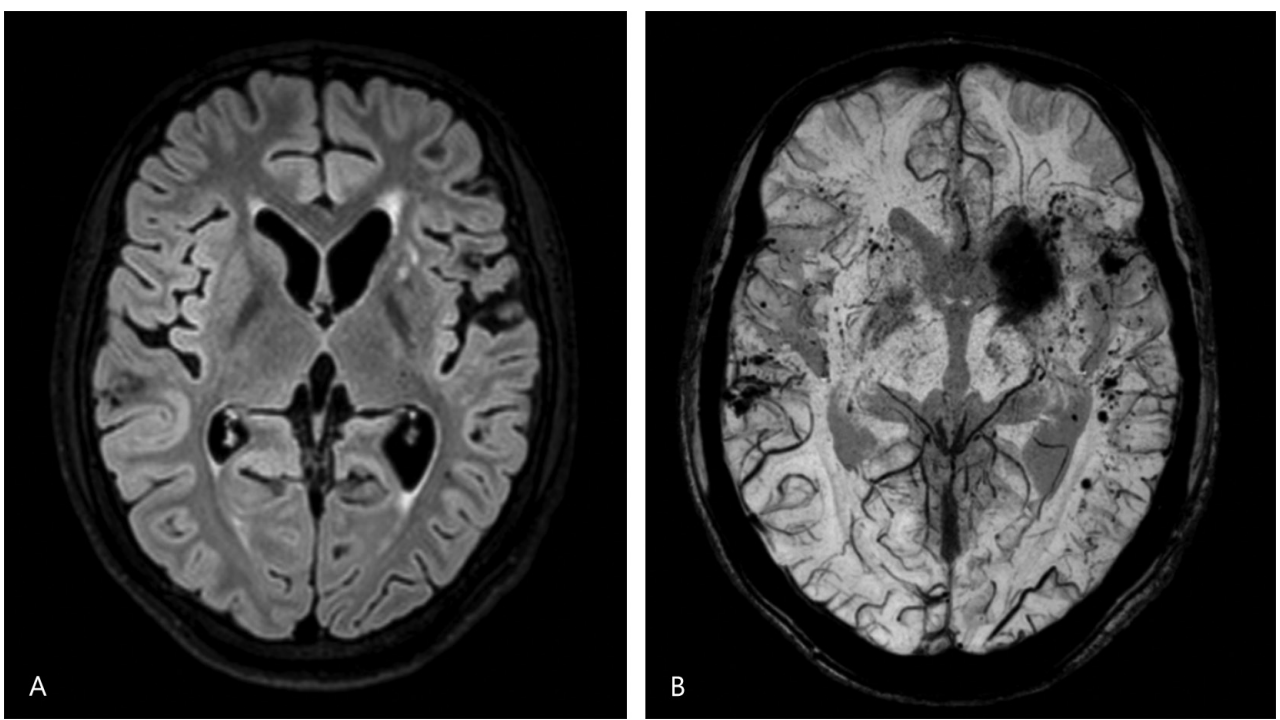

Fig. 1 (A) Magnetic resonance imaging (MRI) brain revealing T2-FLAIR (fluid-attenuated inversion recovery) hyperintensities in the left frontal lobe. (B) Susceptibility-weighted imaging sequence of MRI brain showing multiple microbleeds.
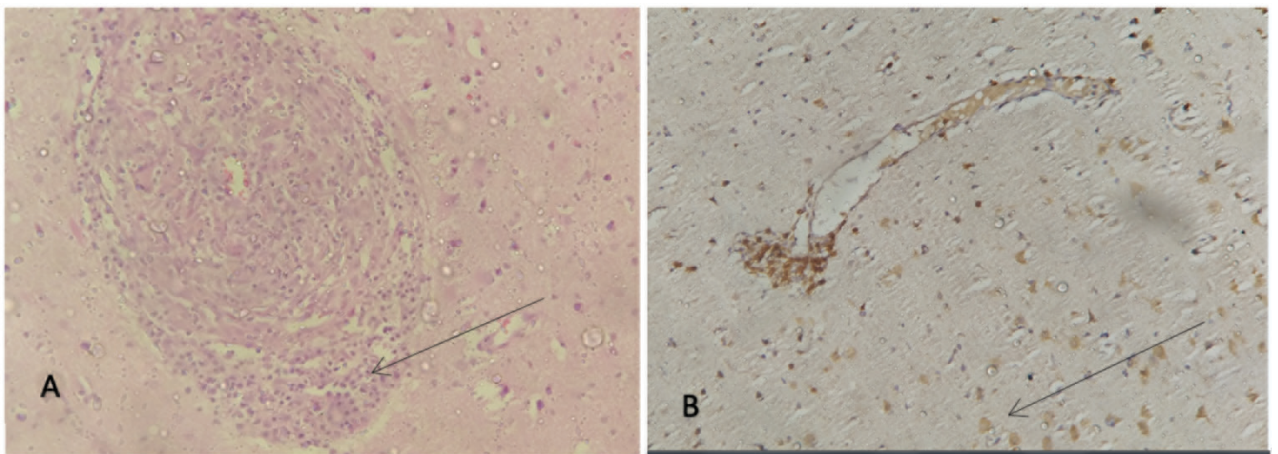

Fig. 2 Right temporal cerebromeningeal biopsy showing chronic perivascular inflammation with predominant CD3+ T-cells with evidence of old hemorrhage, suggestive of granulomatous type primary central nervous system vasculitis on hematoxylin and eosin (A) and immunohistochemistry (B) stains.

\section{Funding}

None.

\section{Conflict of Interest}

None declared.

\section{Acknowledgments}

The authors would like to thank Prof M.V. Padma Srivastava, Prof Mamta B. Singh, Prof. Vinay Goyal, Dr. Ramesh Doddamani and Prof M.C. Sharma for their valuable inputs on this case.

\section{References}

1 Salvarani C, Brown RD Jr., Christianson T, et al. An update of the Mayo Clinic cohort of patients with adult primary central nervous system vasculitis: description of 163 patients. Medicine (Baltimore) 2015;94(21):e738

2 Calabrese LH, Mallek JA. Primary angiitis of the central nervous system. Report of 8 new cases, review of the literature, and proposal for diagnostic criteria. Medicine (Baltimore) 1988; 67(1):20-39

3 Headache Classification Committee of the International Headache Society (IHS) The international classification of headache disorders, 3rd edition. Cephalalgia2018;38(1):1:211

4 Goadsby PJ, Lipton RB, Ferrari MD. Migraine-current understanding and treatment. N Engl J Med 2002;346(4):257-270

5 Boulouis G, de Boysson H, Zuber M, et al; French Vasculitis Group. Primary angiitis of the central nervous system: magnetic resonance imaging spectrum of parenchymal, meningeal, and vascular lesions at baseline. Stroke 2017;48(5):1248-1255 